\title{
THE BAR CONSTRUCTION AND AFFINE STACKS
}

\author{
MARTIN OLSSON
}

\section{INTRODUCTION}

The purpose of this note is to clarify a technical point which arose in our work on nonabelian p-adic Hodge theory in [Ol]. While the main content of this paper is purely algebraic and not concerned with $p$-adic Hodge theory, let us first recall the motivation.

1.1. Let $V$ be a complete discrete valuation ring with perfect residue field of characteristic $p>0$ and fraction field $K$ of characteristic 0 . Fix an algebraic closure $K \subset \bar{K}$. Let $X / V$ be a smooth proper scheme, and let $D \subset X$ be a divisor with normal crossings over $V$. Assume that $X_{\bar{K}}$ is connected. Let $X^{\circ}$ denote $X-D$ and assume given a point $x \in X^{\circ}(V)$. We can then consider the pro-unipotent fundamental group of $\left(X^{\circ}, x\right)$ in the various motivic realizations (Betti, étale, de Rham, and crystalline). The étale realization $\pi_{1}^{\text {et }}\left(X_{\bar{K}}^{\circ}, x\right)$ is the pro-unipotent group scheme over $\mathbb{Q}_{p}$ which is Tannaka dual to the category of unipotent $\mathbb{Q}_{p^{-}}$ local systems on the geometric generic fiber $X_{\bar{K}}^{\circ}$. There is a natural action of the Galois group $G_{K}:=\operatorname{Gal}(\bar{K} / K)$ on $\pi_{1}^{\text {et }}\left(X_{\bar{K}}^{\circ}, x\right)$, and it is shown in $[\mathrm{Ol}]$ that the coordinate $\operatorname{ring} \mathscr{O}_{\pi_{1}^{\text {et }}\left(X_{\bar{K}}^{\circ}, x\right)}$ is an ind-crystalline representation (that is, a direct limit of crystalline representations).

Let us review what this means (see $[\mathrm{Fo}]$ for more details). Let $W$ denote the ring of Witt vectors of the residue field of $V$, and let $K_{0} \subset K$ denote the field of fractions of $W$. Let $\sigma: W \rightarrow W$ be the canonical lifting of Frobenius, and write also $\sigma$ for the induced automorphism of $K_{0}$. Let $\mathrm{B}_{\text {cris }}(V)$ denote Fontaine's $K_{0}$-algebra defined for example in [Fo]. Recall that $\mathrm{B}_{\text {cris }}(V)$ comes equipped with an action of $G_{K}$, and a semi-linear automorphism $\varphi$. The ring $\mathrm{B}_{\text {cris }}(V)$ is contained in a $K$-algebra $\mathrm{B}_{\mathrm{dR}}(V)$ which is a discrete valuation field. In particular, the valuation on $\mathrm{B}_{\mathrm{dR}}(V)$ defines a filtration on $\mathrm{B}_{\mathrm{dR}}(V)$. For a continuous finite dimensional $G_{K}$-representation $M$ over $\mathbb{Q}_{p}$, define

$$
\mathbf{D}(M):=\left(M \otimes_{\mathbb{Q}_{p}} \mathrm{~B}_{\text {cris }}(V)\right)^{G_{K}},
$$

where the action of $G_{K}$ on $M \otimes_{\mathbb{Q}_{p}} \mathrm{~B}_{\text {cris }}(V)$ is the diagonal action. Then $\mathbf{D}(M)$ is a finite dimensional $K_{0}$-vector space with a semilinear automorphism induced by the automorphism of $\mathrm{B}_{\text {cris }}(V)$. There is a natural map

$$
\alpha_{M}: \mathbf{D}(M) \otimes_{K_{0}} \mathrm{~B}_{\text {cris }}(V) \rightarrow M \otimes_{\mathbb{Q}_{p}} \mathrm{~B}_{\text {cris }}(V)
$$

which is always injective. The representation $M$ is called crystalline if $\alpha_{M}$ is an isomorphism. In this case we obtain an isomorphism

$$
\mathbf{D}(M) \otimes_{K_{0}} \mathrm{~B}_{\mathrm{dR}}(V) \simeq M \otimes_{\mathbb{Q}_{p}} \mathrm{~B}_{\mathrm{dR}}(V),
$$

which induces a filtration on $\mathbf{D}(M) \otimes_{K_{0}} K$. 
1.2. It is shown in $[\mathrm{Ol}]$ that there is a canonical isomorphism of Hopf-algebras over $K_{0}$

$$
\mathrm{D}\left(\mathscr{O}_{\pi_{1}^{\text {et }}\left(X_{\bar{K}}^{\circ}, x\right)}\right) \simeq \mathscr{O}_{\pi_{1}^{\text {crys }}\left(X^{\circ}, x\right)}
$$

where $\pi_{1}^{\text {crys }}\left(X^{\circ}, x\right)$ denotes the Tannaka dual of the category of unipotent log isocrystals on $(X, D) / W$. This isomorphism is compatible with the Frobenius automorphisms, where the Frobenius automorphism on $\pi_{1}^{\text {crys }}\left(X^{\circ}, x\right)$ is induced by Frobenius pullback on the category of unipotent log isocrystals.

We also have an isomorphism

$$
\pi_{1}^{\text {crys }}\left(X^{\circ}, x\right) \otimes_{K_{0}} K \simeq \pi_{1}^{\mathrm{dR}}\left(X_{K}^{\circ}, x\right),
$$

where $\pi_{1}^{\mathrm{dR}}\left(X_{K}^{\circ}, x\right)$ is the Tannaka dual of the category of unipotent modules with integrable connection on $X_{K}^{\circ}$. In particular, the filtration on $\mathbf{D}\left(\mathscr{O}_{\pi_{1}^{\text {et }}\left(X_{\bar{K}}^{\circ}, x\right)}\right) \otimes_{K_{0}} K$, obtained as above, induces a filtration on $\mathscr{O}_{\pi_{1}^{\mathrm{dR}}\left(X_{K}^{\circ}, x\right)}$.

1.3. On the other hand, Wojtkowiak has defined in [Wo] another filtration on $\mathscr{O}_{\pi_{1}^{\mathrm{dR}}\left(X_{K}^{\circ}, x\right)}$ using the bar construction. The main aim of this note is to show that these two filtrations agree. We also treat the case of spaces of unipotent paths between two different points of $X^{\circ}$.

The motivation for this verification comes from the work of Kim [Ki1, Ki2, Ki3]. In this work, one needs the p-adic Hodge theory of the fundamental group developed in [Ol], but Wojtkowiak's definition of the Hodge filtration is the one used. The complete some of the proofs in Kim's papers, it therefore becomes necessary to know the equality of the two filtrations.

1.4. The verification that the two filtrations agree reduces to an abstract statement about

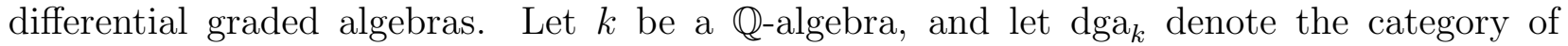
commutative differential graded algebras over $k$. Given an augmented object $\epsilon: A \rightarrow k$ of $\operatorname{dga}_{k}$ there are at least two ways of producing a pro-unipotent group scheme from $(A, \epsilon)$.

The first is to use the bar construction as explained for example in [Wo]. This construction produces a simplicial dga $T(A, \epsilon)$. One can then take the total dga of this simplicial dga and then

$$
B(A, \epsilon):=H^{0}(\operatorname{tot}(T(A)))
$$

has a natural Hopf algebra structure whose associated group scheme we denote by $\pi_{1}^{\text {bar }}(A, \epsilon)$.

The second approach is using Toen's theory of affine stacks [To]. Here one first converts $(A, \epsilon)$ into an augmented cosimplicial $k$-algebra $\epsilon^{\prime}: A^{\prime} \rightarrow k$, and then applies a suitable derived version of the functor $\operatorname{Spec}(-)$ to get a pointed simplicial presheaf $\mathbb{R} \operatorname{Spec}(A)$ on the category of $k$-algebras. One can then take the sheaf associated to the presheaf

$$
R \mapsto \pi_{1}(|\mathbb{R S p e c}(A)(R)|, *)
$$

which by Toen's theory is representable by a pro-unipotent group scheme $\pi_{1}^{\text {aff }}(\mathbb{R} \operatorname{Spec}(A), *)$. Our main purpose in this paper is to establish a natural isomorphism

$$
\pi_{1}^{\mathrm{bar}}(A, \epsilon) \simeq \pi_{1}^{\mathrm{aff}}(\mathbb{R} \operatorname{Spec}(A), *)
$$

as well as a generalization to spaces of paths, and then use this isomorphism to establish the equality of the two filtrations described above. 
1.5. Acknowledgements. We are grateful to Minhyong Kim for stressing the importance of verifying the compatibility of the $p$-adic comparison isomorphism with the bar construction, and to Richard Hain for helpful correspondence.

The author was partially supported by NSF grant DMS-0714086, NSF CAREER grant DMS-0748718, and an Alfred P. Sloan Research Fellowship.

Notation 1.6. For a category $\mathscr{C}$, we write $\mathscr{C}^{\circ}$ for the opposite category. If $\mathscr{C}$ and $\mathscr{D}$ are categories, we write $\mathscr{C}^{\mathscr{D}}$ for the category of functors

$$
\mathscr{D} \rightarrow \mathscr{C} .
$$

We denote the category of simplicial sets by sSet. For a $\mathbb{Q}$-algebra $k$ we write $\operatorname{SPr}(k)$ for the category of simplicial presheaves on the category $\mathrm{Aff}_{k}$ of affine $k$-schemes. So, the category $\operatorname{SPr}(k)$ is the category of functors

$$
\mathrm{Aff}_{k}^{o} \rightarrow s \text { Set. }
$$

For a ring $k$, we denote by dga $a_{k}$ the category of commutative, $\mathbb{Z}$-graded, differential graded algebras, and by $\operatorname{dga}_{\bar{k}} \geq 0$ the full subcategory of differential graded algebras $A$ with $A^{i}=0$ for $i<0$.

We write $C(k)$ for the category of complexes of $k$-modules (possibly unbounded in both directions). By [Hi, 2.2.1] (applied to the identity functor $C(k) \rightarrow C(k)$ ) there is a model category structure on $C(k)$ in which equivalences are quasi-isomorphisms, and fibrations are level-wise surjections.

The standard simplicial category is denoted $\Delta$. The objects of $\Delta$ are finite ordered sets, and morphisms are order preserving maps.

\section{The Model CATEgory StRuCture ON $\mathrm{dga}_{k}$}

The main reference for this section is the paper [Hi].

2.1. Fix a ring $k$. If $A \in \operatorname{dga}_{k}$ is a differential graded $k$-algebra, we write $A^{\sharp}$ for the underlying complex of $k$-modules. The functor

$$
\sharp: \operatorname{dga}_{k} \rightarrow C(k)
$$

has a left adjoint

$$
F: C(k) \rightarrow \text { dga }_{k},
$$

sending a complex $M$ to the free differential graded algebra generated by $M$ (see for example $[\mathrm{Be}, \S 1.2])$. If $M$ is a complex with zero differentials, we write also

$$
\text { Sym } M
$$

for $F(M)$. By the construction in loc. cit., there is a natural direct sum decomposition

$$
\operatorname{Sym}^{\circ} M=\oplus_{k \in \mathbb{Z}} \operatorname{Sym}^{k} M .
$$

If $M=N[-i]$ for some $k$-module $M$, then $\operatorname{Sym} M$ is the symmetric algebra on $N$ if $i$ is even, and the exterior algebra on $N$ if $i$ is odd (and $N$ is placed in degree $i$ ). 
2.2. Define a morphism $f: A \rightarrow B$ in dga $_{k}$ to be an equivalence (resp. fibration) if the underlying morphism of complexes $A^{\sharp} \rightarrow B^{\sharp}$ is a quasi-isomorphism (resp. if $A^{i} \rightarrow B^{i}$ is surjective for every $i$ ).

As explained in $[\mathrm{Hi}, 2.2 .1]$ there is a model category structure on dga $\mathrm{a}_{k}$ with the above defined equivalences and fibrations, and cofibrations defined by the left lifting property with respect to trivial fibrations.

2.3. In fact in $[\mathrm{Hi}, 2.2 .3]$ there is also a description of cofibrations in $\operatorname{dga}_{k}$. Let $M \in C(k)$ be a complex of free $k$-modules with zero differentials, and let $\alpha: M \rightarrow A^{\sharp}$ be a morphism for some $A \in \operatorname{dga}_{k}$. We can then form a morphism

$$
A \rightarrow A\langle M, \alpha\rangle
$$

as follows. The underlying algebra of $A\langle M, \alpha\rangle$ is the tensor product

$$
A \otimes_{k} \operatorname{Sym}(M[1]),
$$

with the differential given by the differential on $A$, and such that

$$
\left.d\right|_{M}=\left.\alpha\right|_{M}
$$

Then $A \rightarrow A\langle M, \alpha\rangle$ is a cofibration. We call a morphism $A \rightarrow A\langle M, \alpha\rangle$ obtained in this way an elementary cofibration. We say that a map $A \rightarrow B$ is a standard cofibration if it is a limit of a sequence

$$
A=A_{1} \rightarrow A_{2} \rightarrow A_{3} \rightarrow \cdots
$$

where each $A_{i} \rightarrow A_{i+1}$ is an elementary cofibration. By [Hi, 2.2.5] every cofibration in dga ${ }_{k}$ is a retract of a standard cofibration.

2.4. For $A \in \operatorname{dga}_{k}$, let $\operatorname{Mod}_{A}$ denote the category of differential graded $A$-modules. As in $[\mathrm{Hi}, 3.1]$, the category $\operatorname{Mod}_{A}$ has a model category structure in which a morphism $M \rightarrow N$ in $\operatorname{Mod}_{A}$ is an equivalence (resp. fibration) if the underlying morphism in $C(k)$ is an equivalence (resp. fibration). For $M \in \operatorname{Mod}_{A}$ we write $M^{\sharp}$ for the underlying object of $C(k)$.

Cofibrations in $\operatorname{Mod}_{A}$ can also be described using the general recipe in [Hi, 2.2.4]. Let $M \in \operatorname{Mod}_{A}$ be an $A$-module, $N \in C(k)$ a complex of free $k$-modules with zero differentials, and $\beta: N \rightarrow M^{\sharp}$ a morphism in $C(k)$. We define

$$
M\{N, \beta\}
$$

to be the object of $\operatorname{Mod}_{A}$ with underlying $A$-module

$$
M \oplus\left(A \otimes_{k} N[1]\right)
$$

and differential given by the differential on $M$ and the map $\beta$ on $N$. We say that a morphism $M \rightarrow M^{\prime}$ in $\operatorname{Mod}_{A}$ is an elementary cofibration if $M^{\prime} \simeq M\{N, \beta\}$ for some pair $(N$, $\beta)$, where $N$ is a complex of free modules with zero differentials, and $\beta: N \rightarrow M^{\sharp}$ is a morphism of complexes. A morphism $M \rightarrow M^{\prime}$ is called a standard cofibration if it is the limit of a sequence

$$
M=M_{0} \rightarrow M_{1} \rightarrow M_{2} \rightarrow \cdots,
$$

where each $M_{i} \rightarrow M_{i+1}$ is an elementary cofibration. By [Hi, 2.2.5] every cofibration in $\operatorname{Mod}_{A}$ is a retract of an elementary cofibration. 
Lemma 2.5. Let $M \in \operatorname{Mod}_{A}$ be a cofibrant object. Then the functor

$$
M \otimes_{A}(-): \operatorname{Mod}_{A} \rightarrow C(k)
$$

takes equivalences to equivalences.

Proof. Since any cofibration is a retract of a standard cofibration, it suffices to consider the case when $M$ is the limit of a sequence

$$
0=M_{0} \rightarrow M_{1} \rightarrow M_{2} \rightarrow \cdots,
$$

where each $M_{i} \rightarrow M_{i+1}$ is an elementary cofibration. Since a filtering direct limit of equivalences is an equivalence, it therefore suffices by induction to prove that if $M \in \operatorname{Mod}_{A}$ is an object such that $M \otimes_{A}(-)$ takes equivalences to equivalences, then for an elementary cofibration

$$
M \rightarrow M\{N, \beta\}
$$

the functor $M\{N, \beta\} \otimes_{A}(-)$ takes equivalences to equivalences. For this note that if $S \in$ $\operatorname{Mod}_{A}$, then

$$
M\{N, \beta\} \otimes_{A} S \simeq\left(M \otimes_{A} S\right) \oplus\left(N[1] \otimes_{k} S\right)
$$

with differential induced by the map $\beta$. That is, we have an isomorphism in $C(k)$

$$
M\{N, \beta\} \otimes_{A} S \simeq \operatorname{Cone}\left(\beta: N \otimes_{k} S \rightarrow M \otimes_{A} S\right) .
$$

It follows that it suffices to show that the functor $(-) \otimes_{k} N$ takes equivalences to equivalences, which is immediate as each $N^{i}$ is a free $k$-module.

2.6. Let $\operatorname{dga}_{k, A}$ denote the category of morphisms $A \rightarrow B$ in $\operatorname{dga}_{k}$. There is a natural forgetful functor

$$
\operatorname{dga}_{k, A} \rightarrow \operatorname{Mod}_{A}, \quad(A \rightarrow B) \mapsto B^{\dagger},
$$

where $B^{\dagger}$ denotes the underlying differential graded $A$-module of $B$.

Proposition 2.7. For any cofibration $A \rightarrow B$ in $\mathrm{dga}_{k}$, the $\operatorname{module} B^{\dagger} \in \operatorname{Mod}_{A}$ is cofibrant.

Proof. By [Hi, 2.2.5], the map $A \rightarrow B$ is a retract of a standard cofibration, so it suffices to consider the case when $A \rightarrow B$ is a standard cofibration. By induction it therefore suffices to show that if $A^{\prime} \in \operatorname{dga}_{k, A}, M \in C(k)$ is a complex of free $k$-modules with zero differential, and $\alpha: M \rightarrow\left(A^{\prime}\right)^{\sharp}$ is a morphism in $C(k)$, then the induced map

$$
\left(A^{\prime}\right)^{\dagger} \rightarrow\left(A^{\prime}\langle M, \alpha\rangle\right)^{\dagger}
$$

is a cofibration in $\operatorname{Mod}_{A}$.

Note that $A^{\prime}\langle M, \alpha\rangle$ is an inductive limit of a sequence

$$
A^{\prime} \rightarrow A_{1}^{\prime} \rightarrow A_{2}^{\prime} \rightarrow \cdots
$$

where $A_{i}^{\prime} \rightarrow A_{i+1}^{\prime}$ is of the form $A_{i}^{\prime} \rightarrow A_{i}^{\prime}\left\langle M_{i}, \alpha_{i}\right\rangle$ for some $M_{i}=N[-i]$ with $N$ a free $k$-module. Therefore we may further assume that $M=N[-i]$ for some free $k$-module $N$.

For $n \geq 0$ define an object of $\operatorname{Mod}_{A}$

$$
\Sigma^{(n)}:=\oplus_{k=0}^{n} A^{\prime, \dagger} \otimes_{k} \operatorname{Sym}^{k}(N[-i]),
$$

with differential given by the differential on $A^{\prime, \dagger}$ and the map

$$
A^{\prime, \dagger} \otimes_{k} \operatorname{Sym}^{k}(N[-i]) \rightarrow A^{\prime, \dagger} \otimes_{k} \operatorname{Sym}^{k-1}(N[-i])
$$


induced by the map $\alpha$. We then have a sequence in $\operatorname{Mod}_{A}$ with limit $A^{\prime, \dagger} \rightarrow\left(A^{\prime}\langle M, \alpha\rangle\right)^{\dagger}$

$$
A^{\prime, \dagger}=\Sigma^{(0)} \rightarrow \Sigma^{(1)} \rightarrow \Sigma^{(2)} \rightarrow \cdots
$$

where each $\Sigma^{(i)} \rightarrow \Sigma^{(i+1)}$ is a standard cofibration. It follows that $A^{\prime, \dagger} \rightarrow\left(A^{\prime}\langle M, \alpha\rangle\right)^{\dagger}$ is also a cofibration.

Corollary 2.8. Consider a diagram in dga $_{k}$

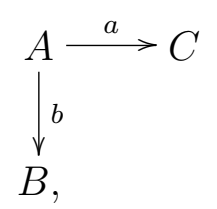

where $a$ is a cofibration. Then $B \otimes_{A} C$ represents the homotopy colimit in $\mathrm{dga}_{k}$ of the diagram 2.8.1.

Proof. By 2.7, the object $C^{\dagger} \in \operatorname{Mod}_{A}$ is cofibrant, and therefore the functor

$$
(-) \otimes_{A} C^{\dagger}: \operatorname{Mod}_{A} \rightarrow C(k)
$$

takes equivalences to equivalences. In particular, given a factorization of $b$

$$
A \stackrel{c}{\longrightarrow} E \stackrel{d}{\longrightarrow} B
$$

where $c$ is a cofibration and $d$ is an equivalence, the induced map

$$
E \otimes_{A} C \rightarrow B \otimes_{A} C
$$

is an equivalence.

2.9. For $M \in \operatorname{Mod}_{A}$, we can also compute the derived functor

$$
M \otimes_{A}^{\mathbb{L}}(-): \operatorname{Ho}\left(\operatorname{Mod}_{A}\right) \rightarrow D(k)
$$

as follows (the following was also remarked in [Hi, §3.4]).

For a simplicial object $P . \in \operatorname{Mod}_{A}^{\Delta^{o}}$, let tot $(P$.) denote the (sum) total complex defined by

$$
(\operatorname{tot}(P .))^{n}:=\oplus_{i+j=n} P_{-i}^{j}
$$

and the standard differential induced by the simplicial structure (see for example [Il, I.1.2.1]). The complex $\operatorname{tot}(P$. $)$ is naturally an object of $\operatorname{Mod}_{A}$ with $a \in A^{k}$ acting by the given multiplication maps

$$
a \cdot(-): P_{-i}^{j} \rightarrow P_{-i}^{j+k}
$$

Proposition 2.10. Assume P. $\in \operatorname{Mod}^{\Delta^{o}}$ is a simplicial A-module, with each $P_{i}$ cofibrant. Then the functor

$$
\operatorname{tot}(P .) \otimes_{A}(-): \operatorname{Mod}_{A} \rightarrow C(k)
$$

preserves arbitrary equivalences. In particular, for any $M \in \operatorname{Mod}_{A}$ the $\operatorname{complex} \operatorname{tot}(P$. $) \otimes_{A} M \in$ $C(k)$ represents tot $(P.) \otimes_{A}^{\mathbb{L}} M \in D(k)$. 
Proof. Note first that if $P . \rightarrow P^{\prime}$. is a morphism in $\operatorname{Mod}_{A}^{\Delta^{o}}$ such that the map $P_{i} \rightarrow P_{i}^{\prime}$ is an equivalence for all $i$, then the induced map

$$
\operatorname{tot}(P .) \rightarrow \operatorname{tot}\left(P_{.}^{\prime}\right)
$$

is also an equivalence. Furthermore, by construction we have

$$
\operatorname{tot}(P .) \otimes_{A} M \simeq \operatorname{tot}\left(P . \otimes_{A} M\right) .
$$

If $M \rightarrow M^{\prime}$ is an equivalence, we therefore get that the map

$$
\operatorname{tot}(P .) \otimes_{A} M \simeq \operatorname{tot}\left(P . \otimes_{A} M\right) \rightarrow \operatorname{tot}\left(P . \otimes_{A} M^{\prime}\right) \simeq \operatorname{tot}(P .) \otimes_{A} M^{\prime}
$$

is an equivalence.

2.11. If $P . \in \operatorname{dga}_{k}^{\Delta^{o}}$ is a simplicial object in $\operatorname{dga}_{k}$, then the total complex $\operatorname{tot}\left(P^{\dagger}\right)$ has a natural structure of an object of $\operatorname{dga}_{k}$, which we will denote by $\operatorname{tot}(P$.). The multiplication map is given by the shuffle product (see for example [Wo, §2]).

Corollary 2.12. Suppose $P . \in \mathrm{dga}_{k, A}^{\Delta^{o}}$ for some $A \in \mathrm{dga}_{k}$, and that for every $i$ the underlying object $P_{i}^{\dagger} \in \operatorname{Mod}_{A}$ is cofibrant. Then for any morphism $A \rightarrow B$ the homotopy colimit in dga ${ }_{k}$ of the diagram

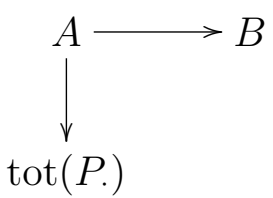

is represented by $\operatorname{tot}(P.) \otimes_{A} B$.

Proof. Indeed it suffices to verify that $(\operatorname{tot}(P .))^{\dagger} \otimes_{A} B^{\dagger} \in C(k)$ represents $(\operatorname{tot}(P .))^{\dagger} \otimes_{A}^{\mathbb{L}} B^{\dagger}$ which follows from 2.10.

\section{Filtered Thom-Sullivan cochains}

The content of this section is essentially contained in $[\mathrm{N}-\mathrm{A}]$.

3.1. Let $k$ be a $\mathbb{Q}$-algebra.

For an object $A \in \operatorname{dga}_{k}$, a filtration on $A$ is a decreasing filtration $F$ on the underlying complex of $A$ such that for every $i, j \in \mathbb{Z}$ we have

$$
A^{i} \cdot F^{j} \subset F^{i+j} .
$$

A morphism $(A, F) \rightarrow\left(A^{\prime}, F^{\prime}\right)$ of filtered differential graded algebras is a morphism $f$ :

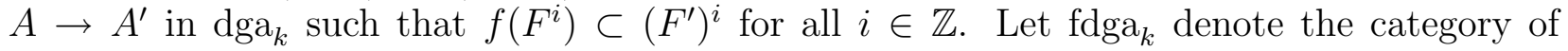
filtered differential graded $k$-algebras. A morphism $f:(A, F) \rightarrow\left(A^{\prime}, F^{\prime}\right)$ is a filtered quasiisomorphism if $A \rightarrow A^{\prime}$ is an equivalence and if for every $i \in \mathbb{Z}$ the map $F^{i} \rightarrow F^{\prime i}$ is a quasi-isomorphism.

Let $\mathrm{Ho}\left(\mathrm{fdga}_{k}\right)$ be the category obtained by inverting filtered quasi-isomorphisms.

The functor of Thom-Sullivan cochains, discussed in [Ol, §2], can be extended to the filtereted context as follows. 
3.2. Let us begin by recalling the definition of the functor of Thom-Sullivan cochains. For $p \geq 0$, let $\nabla(p, \bullet) \in$ dga $_{k}$ denote the de Rham complex of

$$
\operatorname{Spec}\left(k\left[t_{0}, \ldots, t_{p}\right] /\left(\sum_{i=0}^{p} t_{i}=1\right)\right)
$$

over $k$. As explained in [Ol, 2.13], as $p$ varies we obtain a simplicial differential graded algebra

$$
\Omega: \Delta^{o} \rightarrow \operatorname{dga}_{k}, \quad[p] \mapsto \nabla(p, \bullet)
$$

Let $\mathcal{M}_{\Delta}$ be the category whose objects are morphisms $f:[i] \rightarrow[j]$ in $\Delta$, and for which a morphism

$$
(f:[i] \rightarrow[j]) \rightarrow\left(g:\left[i^{\prime}\right] \rightarrow\left[j^{\prime}\right]\right)
$$

is a commutative diagram in $\Delta$

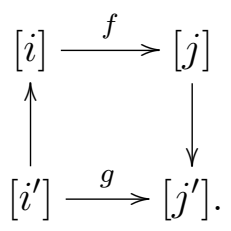

If $A . \in \operatorname{dga}_{k}^{\Delta}$ is a cosimplicial differential graded algebra, we define

$$
\text { A. } \otimes \Omega: \mathcal{M}_{\Delta} \rightarrow \operatorname{dga}_{k}, \quad(f:[i] \rightarrow[j]) \mapsto A_{i} \otimes \Omega_{j} .
$$

The functor of Thom-Sullivan cochains

$$
T: \operatorname{dga}_{k}^{\Delta} \rightarrow \operatorname{dga}_{k}
$$

is defined by

$$
T(A):=\lim _{\overleftarrow{\mathcal{M}_{\Delta}}} A . \otimes \Omega
$$

3.3. This construction also extends to the filtered context. View $\nabla(p, \bullet)$ as an object of fdga with filtration given by

$$
F^{i} \nabla(p, \bullet):= \begin{cases}\nabla(p, \bullet) & \text { if } i \leq 0 \\ 0 & \text { if } i>0\end{cases}
$$

With this definition the transition maps for $\Omega$ are compatible with the filtrations, so we obtain a functor

$$
\Omega^{f}: \Delta^{o} \rightarrow \mathrm{fdga}_{k} .
$$

Furthermore, for a cosimplicial object $A . \in \operatorname{fdga}_{k}^{\Delta}$ we obtain a functor

$$
\text { A. } \otimes \Omega^{f}: \mathcal{M}_{\Delta} \rightarrow \mathrm{fdga}_{k}
$$

and we define

$$
T^{f}(A .):=\lim _{\overleftarrow{\mathcal{M}_{\Delta}}} A . \otimes \Omega^{f} \in \mathrm{fdga}_{k}
$$

As explained in [N-A, 6.3], integration defines a natural filtered quasi-isomorphism of filtered complexes

$$
\int: T^{f}(A .) \rightarrow \operatorname{Tot}(A .)
$$


In particular, $T^{f}$ passes to a functor

$$
T^{f}: \operatorname{Ho}\left(\mathrm{fdga}_{k}^{\Delta}\right) \rightarrow \mathrm{Ho}\left(\mathrm{fdga}_{k}\right) .
$$

\section{THE BAR CONSTRUCTION AND DERIVED TENSOR PRODUCT}

4.1. Let $A \in \operatorname{dga}_{k}$ be a cofibrant object with two augmentations $x, y: A \rightarrow k$. The bar construction provides a representative for the homotopy pushout of the diagram (which will be of special importance for spaces of paths)

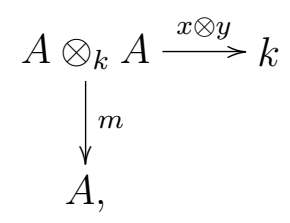

where $m$ denotes the multiplication map.

4.2. Let $\mathscr{C}$ and $\mathscr{D}$ be categories, and let

$$
U: \mathscr{C} \rightarrow \mathscr{D}, \quad T: \mathscr{D} \rightarrow \mathscr{C}
$$

be functors with $T$ left adjoint to $U$. Write

$$
a: \mathrm{id}_{\mathscr{D}} \rightarrow U T, \quad b: T U \rightarrow \mathrm{id}_{\mathscr{C}}
$$

for the adjunction morphisms. Given an object $X \in \mathscr{C}$, we can form its canonical simplicial resolution $(T, U) .(X) \in \mathscr{C}^{\Delta^{o}}$ as follows (for more details see [Il, I, §1.5]).

Define

$$
(T, U)_{n}(X):=(T U)^{[n]}(X),
$$

where we write $(T U)^{[n]}$ for the $n+1$-fold composite

$$
(T U) \circ(T U) \circ \cdots \circ(T U) .
$$

For $i=0, \ldots, n+1$, let

$$
d_{i}:(T, U)_{n+1}(X) \rightarrow(T, U)_{n}(X)
$$

be the map induced by the morphism of functors

$$
\operatorname{id}_{(T U)^{[0, i-1]}} \circ b \circ \operatorname{id}_{(T U)^{[i+1, n]}},
$$

and for $i=0, \ldots, n$, let

$$
s_{i}:(T, U)_{n}(X) \rightarrow(T, U)_{n+1}(X)
$$

be the map induced by the morphism of functors

$$
\operatorname{id}_{(T U)^{[0, i-1]}} \circ \operatorname{id}_{T} \circ a \circ \operatorname{id}_{U} \circ \operatorname{id}_{(T U)^{[i+1, n]}} .
$$

We then obtain a simplicial object in $\mathscr{C}$ denoted by $(T, U) .(X)$.

The map $b$ induces a natural augmentation

$$
\pi:(T, U) \cdot(X) \rightarrow X
$$


4.3. We will apply this construction as follows. Let $A \rightarrow A^{\prime}$ be a morphism in $\operatorname{dga}_{k}$. We can then consider the restriction functor

$$
U: \operatorname{dga}_{k, A^{\prime} /} \rightarrow \operatorname{dga}_{k, A /}
$$

which has a left adjoint

$$
A^{\prime} \otimes_{A}(-): \operatorname{dga}_{k, A /} \rightarrow \operatorname{dga}_{k, A^{\prime} /}
$$

Lemma 4.4. For any object $B \in \operatorname{dga}_{k, A^{\prime} /}$ the map in $\operatorname{dga}_{k, A^{\prime} /}$

$$
\operatorname{tot}((T, U) .(B)) \rightarrow B
$$

induced by the augmentation $(T, U) .(B) \rightarrow B$, is an equivalence.

Proof. It suffices to verify that the map in $\operatorname{dga}_{k, A}$ obtained by applying the functor $U$ is an equivalence. This follows from [Il, I, 1.5.3 (i)].

Lemma 4.5. Suppose $A \rightarrow B$ and $A \rightarrow A^{\prime}$ are cofibrations. Then for every $n$ the morphism $A^{\prime} \rightarrow(T, U)_{n}(B)$ is a cofibration in $\operatorname{dga}_{k}$.

Proof. We proceed by induction on $n$.

For the case $n=0$, note that $(T, U)_{0}(B)=A^{\prime} \otimes_{A} B$, with $A^{\prime}$-module structure given by the morphism

$$
A^{\prime} \rightarrow A^{\prime} \otimes_{A} B, \quad x \mapsto x \otimes 1 .
$$

Since a pushout of a cofibration is again a cofibration, the assumption that $A \rightarrow B$ is a cofibration implies that $A^{\prime} \rightarrow(T, U)_{0}(B)$ is also a cofibration.

For the inductive step, assume that $A^{\prime} \rightarrow(T, U)_{n-1}(B)$ is a cofibration. Then the composite

$$
A \rightarrow A^{\prime} \rightarrow(T, U)_{n-1}(B)
$$

is also a cofibration, and $(T, U)_{n}(B)$ is the pushout of the diagram

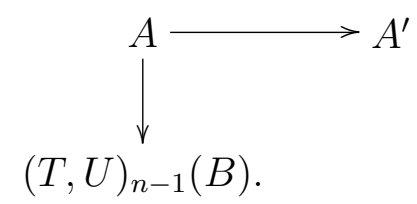

Corollary 4.6. Consider a diagram in $\operatorname{dga}_{k}$

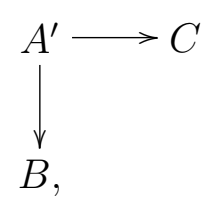

and let $A \rightarrow A^{\prime}$ be a cofibration such that the composite $A \rightarrow A^{\prime} \rightarrow B$ is also a cofibration. Then the homotopy colimit of the diagram 4.6.1 in $\mathrm{dga}_{k}$ is represented by

$$
\operatorname{tot}((T, U) .(B)) \otimes_{A^{\prime}} C \simeq \operatorname{tot}\left((T, U) .(B) \otimes_{A^{\prime}} C\right) .
$$

Proof. Combine 2.7, 2.12, and 4.5. 
4.7. A particular special case is the following. Let $A$ be a cofibrant object in dga with two augmentations $x, y: A \rightarrow k$. Then we can apply the previous corollary to the map

$$
A \rightarrow A \otimes_{k} A, \quad x \mapsto x \otimes 1,
$$

and the diagram

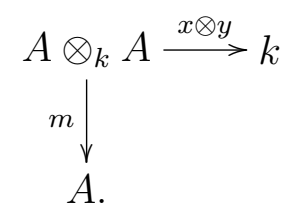

The simplicial differential graded algebra

$$
\operatorname{tot}\left((T, U) .(A) \otimes_{A \otimes A} k\right)
$$

is called the bar complex of $(A, x, y)$, and will be denoted by $B(A, x, y)$ (see for example [Wo, $\S 3])$. It follows from the above discussion that $B(A, x, y)$ represents the homotopy colimit in the category $\operatorname{dga}_{k}$ of the diagram 4.7.1.

The simplicial differential graded algebra

$$
(T, U) .(A) \otimes_{A \otimes A} k
$$

can be described more explicitly as follows (compare with [Wo, §3]). We have

$$
(T, U)_{n}(A) \otimes_{A \otimes A} k=\underbrace{A \otimes_{k} A \otimes \cdots \otimes A}_{n}
$$

and the simplicial structure is given by the maps

$$
\begin{gathered}
d_{n}\left(w_{1} \otimes \cdots \otimes w_{n}\right)=x\left(w_{1}\right) w_{2} \otimes \cdots \otimes w_{n}, \\
d_{n-i}\left(w_{1} \otimes \cdots \otimes w_{n}\right)=w_{1} \otimes \cdots \otimes w_{i} \cdot w_{i+1} \otimes \cdots \otimes w_{n}, \quad 0<i<n, \\
d_{0}\left(w_{1} \otimes \cdots \otimes w_{n}\right)=y\left(w_{n}\right) w_{1} \otimes \cdots \otimes w_{n-1},
\end{gathered}
$$

and

$$
s_{i}\left(w_{1} \otimes \cdots \otimes w_{n}\right)=w_{1} \otimes \cdots \otimes w_{n-i} \otimes 1 \otimes w_{n-i+1} \otimes \cdots \otimes w_{n}, \quad 0 \leq i \leq n .
$$

Remark 4.8. In the case when $k$ is a field, the assumption that $A$ is cofibrant in 4.7 is unnecessary. Indeed in this case any complex $M \in C(k)$ is $K$-flat in the sense of [Sp, 5.1]. In particular, if $A^{\prime} \rightarrow A$ is an equivalence in $\operatorname{dga}_{k}$, then the induced maps

$$
\underbrace{A^{\prime} \otimes_{k} A^{\prime} \otimes \cdots \otimes A^{\prime}}_{n} \rightarrow \underbrace{A \otimes_{k} A \otimes \cdots \otimes A}_{n}
$$

are also equivalences, and so the map on bar complexes

$$
B\left(A^{\prime}, x, y\right) \rightarrow B(A, x, y)
$$

is also an equivalence. 


\section{Comparison of $\operatorname{dga}_{k}$ AND dga $\frac{\geq 0}{k}$}

5.1. Let $A \in \operatorname{dga}_{\bar{k}}^{\geq 0}$ be an object with two augmentations $x, y: A \rightarrow k$. Assume that $A$ is cofibrant when viewed as an object of $\operatorname{dga}_{k}$. We can then consider the diagram

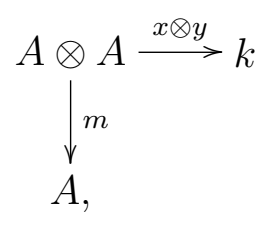

which can be viewed either as a diagram in $\operatorname{dga}_{\bar{k}}^{\geq 0}$ or in $\operatorname{dga}_{k}$. We already know by 4.7 that the homotopy colimit in $\mathrm{dga}_{k}$ is represented by the bar complex $B(A, x, y)$, whereas for the spaces of paths we are interested in the homotopy colimit in the category $\operatorname{dga} \mathrm{k}_{k}^{\geq 0}$.

5.2. Let

$$
J: \operatorname{dga}_{k}^{\geq 0} \rightarrow \operatorname{dga}_{k}
$$

be the inclusion functor. This functor has a left adjoint

$$
\tau_{\geq 0}: \operatorname{dga}_{k} \rightarrow \operatorname{dga}_{k}^{\geq 0},
$$

which sends $A \in \operatorname{dga}_{k}$ to the quotient of $A$ by the differential graded ideal generated by elements $x \in A^{i}$, for $i<0$.

In particular, the forgetful functor

$$
\sharp: \operatorname{dga}_{\bar{k}}^{\geq 0} \rightarrow C(k)
$$

has a left adjoint given by the composite

$$
C(k) \stackrel{F}{\longrightarrow} \operatorname{dga}_{k} \stackrel{\tau \geq 0}{\longrightarrow} \operatorname{dga}_{k} \underset{k}{ } .
$$

A straightforward verification, which we leave to the reader, shows that the assumptions of $[\mathrm{Hi}, 2.2 .1]$ are verified, so we obtain a model category structure on $\mathrm{dga} \mathrm{a}_{k}^{\geq 0}$ in which a morphism $A \rightarrow B$ is an equivalence (resp. fibration) if and only if the induced map $A^{\sharp} \rightarrow B^{\sharp}$ in $C(k)$ is an equivalence (resp. fibration).

5.3. Since the functor $J$ clearly preserves equivalences and fibrations, the functor $\tau_{\geq 0}$ takes cofibrations to cofibrations, and we have derived functors (see for example [Ho, 1.3.4])

$$
\mathbb{L}_{\tau_{\geq 0}}: \operatorname{Ho}\left(\operatorname{dga}_{k}\right) \rightarrow \operatorname{Ho}\left(\operatorname{dga}_{\bar{k}}^{\geq 0}\right), \quad J: \operatorname{Ho}\left(\operatorname{dga}_{\bar{k}}^{\geq 0}\right) \rightarrow \operatorname{Ho}\left(\operatorname{dga}_{k}\right) .
$$

Given a diagram in $\operatorname{dga}_{\bar{k}}^{\geq 0}$

$$
\mathscr{D}: \begin{aligned}
& A \\
& \downarrow \\
& B
\end{aligned}
$$

let $\operatorname{hocolim}(\mathscr{D})\left(\right.$ resp. hocolim $\left.\geq^{\geq 0}(\mathscr{D})\right)$ be the homotopy colimit of $\mathscr{D}$ in $\operatorname{dga}_{k}\left(\right.$ resp. $\left.\operatorname{dga}_{k}^{\geq 0}\right)$. There is then a natural map

$$
\operatorname{hocolim}(\mathscr{D}) \rightarrow J\left(\operatorname{hocolim}^{\geq 0}(\mathscr{D})\right) .
$$


Lemma 5.4. Suppose there exists factorizations in $\operatorname{dga} \underset{k}{\geq 0}$

$$
A \stackrel{a}{\longrightarrow} C^{\prime} \stackrel{c}{\longrightarrow} C
$$

and

$$
A \stackrel{b}{\longrightarrow} B^{\prime} \stackrel{d}{\longrightarrow} B
$$

of $A \rightarrow C$ and $A \rightarrow B$ respectively, such that $c$ and $d$ are equivalences, and $a$ and $b$ define cofibrations in $\mathrm{dga}_{k}$. Then the map 5.3.2 is an isomorphism.

Proof. We may without loss of generality assume that $C=C^{\prime}$ and $B=B^{\prime}$. Then observe that the maps $a$ and $b$ are also cofibrations in $\operatorname{dga}_{k}{ }^{\geq 0}$ as the functor $J$ takes trivial fibrations to trivial fibrations. Therefore both sides of 5.3 .2 are represented by $B \otimes_{A} C$.

\section{SPACES OF PATHS}

6.1. Let $X$ be a simplicial set, and let $x, y: * \rightarrow X$ be two points. The space of paths between $x$ and $y$ can then be constructed as follows.

First we define a cosimplicial object $X^{I}$ in $s$ Set. Namely let $X^{I}$ be the functor

$$
\Delta^{o} \rightarrow s S e t
$$

sending

Note that

$$
[n] \mapsto \prod_{\operatorname{Hom}_{\Delta}([n],[1])} X .
$$

$$
X^{I}([0])=X \times X
$$

so we obtain a diagram in $(s S e t)^{\Delta}$

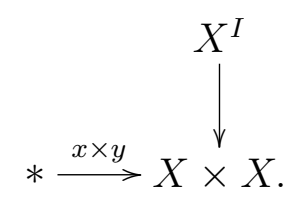

Let $\widetilde{P}_{x, y} \in(s S e t)^{\Delta}$ be the fiber product of this diagram, and define $P_{x, y} \in s$ set to be the homotopy limit over $\Delta$ of $\widetilde{P}_{x, y}$. Then as explained in [Bo, X, 3.3 (i)] the simplicial set $P_{x, y}$ has geometric realization the space of paths between the two points $x$ and $y$ of $|X|$.

6.2. Either projection

$$
X^{I} \longrightarrow X \times X \stackrel{\mathrm{pr}_{i}}{\longrightarrow} X
$$

is a homotopy equivalence. Indeed it suffices to show this after applying the geometric realization functor, in which case it follows from the immediate fact that if $T$ is a topological space then the space of maps $[0,1] \rightarrow T$ is homotopy equivalent to $T$. It follows that the space of paths between $x$ and $y$ is equivalent to the homotopy fiber product of the diagram

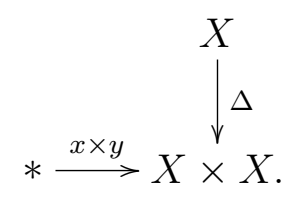


6.3. We can perform a similar construction in $\operatorname{SPr}(k)$, where $k$ is a $\mathbb{Q}$-algebra. Namely, let $\operatorname{SPr}(k)^{\Delta}$ denote the category of cosimplicial objects in $\operatorname{SPr}(k)$. This category has a simplicial model category structure in which cofibrations and equivalences are defined level-wise (see for example [To, §1.2]). If $X \in \operatorname{SPr}(k)$ is a fibrant object and $x, y: * \rightarrow X$ are two points, then as above we can form $X^{I}$, and the fiber product $\widetilde{P}_{x, y} \in \operatorname{SPr}(k)^{\Delta}$. We can then form $P_{x, y}:=\operatorname{holim} \widetilde{P}_{x, y} \in \operatorname{Ho}(\operatorname{SPr}(k))$. By [To, 1.2.1], for every $k$-algebra $R$, the image of $P_{x, y}$ under the evaluation map

$$
\mathrm{Ho}(\operatorname{SPr}(k)) \rightarrow \mathrm{Ho}(s S e t), \quad F \mapsto F(R)
$$

is isomorphic to the space of paths between $x$ and $y$ in $X(R)$.

As above we can also compute the space of paths between $x$ and $y$ as the homotopy fiber product of the diagram

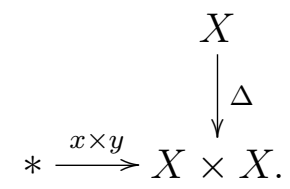

6.4. In the case when $x, y: * \rightarrow X$ is obtained from a cofibrant object $A \in \operatorname{Alg}_{k}^{\Delta}$ with two augmentations $\epsilon_{x}, \epsilon_{y}: A \rightarrow k$, the path space $P_{x, y}$ admits the following algebraic description.

Recall from [To, 2.2.2] that the functor

$$
\operatorname{Spec}(-): \operatorname{Alg}_{k}^{\Delta, o} \rightarrow \operatorname{SPr}(k)
$$

sending $A . \in \operatorname{Alg}_{k}^{\Delta}$ to the simplicial presheaf

$$
B \mapsto\left([n] \mapsto \operatorname{Hom}_{k}\left(A_{n}, B\right)\right)
$$

is a right Quillen functor and has a derived functor

$$
\mathbb{R S p e c}: \operatorname{Ho}\left(\operatorname{Alg}_{k}^{\Delta}\right)^{o} \rightarrow \operatorname{Ho}(\operatorname{SPr}(k)) .
$$

The functor $\mathbb{R S p e c}(-)$ preserves homotopy limits (being a right Quillen functor), and therefore it follows that the path space is given by applying $\mathbb{R} \operatorname{Spec}(-)$ to the homotopy pushout of the diagram in $\operatorname{Alg}_{k}^{\Delta}$

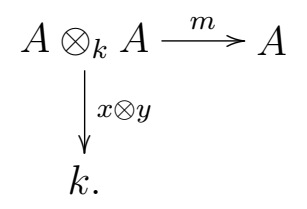

6.5. If $D$ denotes the differential graded algebra obtained from $A$ using the functor of ThomSullivan cochains, and if we still write $x, y: D \rightarrow k$ for the augmentations, then it follows that the differential graded algebra corresponding to $P_{x, y}$ is given by the homotopy pushout, in the category $\operatorname{dga}_{k}^{\geq 0}$, of the diagram

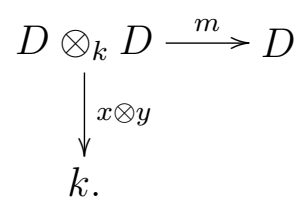


6.6. For $F \in \operatorname{SPr}(k)$, let $\pi_{0}^{\mathrm{sh}}(F)$ denote the sheaf associated to the presheaf

$$
R \mapsto \pi_{0}(X(R)) .
$$

Let $\operatorname{Sh}(k)$ denote the category of sheaves on $\mathrm{Aff}_{k}$, and let

$$
j: \operatorname{Sh}(k) \hookrightarrow \operatorname{SPr}(k)
$$

be the inclusion, sending a sheaf $\mathcal{F}$ to the constant simplicial sheaf

$$
[n] \mapsto \mathcal{F} .
$$

The functor $j$ is right adjoint to the functor $\pi_{0}^{\mathrm{sh}}(-)$.

Lemma 6.7. If $A \in \operatorname{Alg}_{k}^{\Delta}$ is an object such that the sheaf $\pi_{0}^{\mathrm{sh}}(\mathbb{R} \operatorname{Spec}(A))$ is representable, then $\pi_{0}^{\mathrm{sh}}(\mathbb{R} \operatorname{Spec}(A))$ is represented by $\operatorname{Spec}\left(H^{0}(A)\right)$.

Proof. If we view $\mathrm{Sh}(k)$ as a model category in which cofibrations are inclusions, fibrations are epimorphisms, and equivalences are isomorphisms, then the projection $\mathrm{Sh}(k) \rightarrow \mathrm{Ho}(\mathrm{Sh}(k))$ is an equivalence of categories, and the functor $j$ is a right Quillen functor. Note also that $\pi_{0}^{\text {sh }}(-)$ preserves arbitrary equivalences. We therefore obtain that for any $\mathcal{F} \in \operatorname{Sh}(k)$ and $\mathcal{G} . \in \operatorname{Ho}(\operatorname{SPr}(k))$ there is a natural isomorphism

$$
\operatorname{Hom}_{\mathrm{Sh}(k)}\left(\pi_{0}^{\mathrm{sh}}(G .), \mathcal{F}\right) \simeq \operatorname{Hom}_{\mathrm{Ho}(\operatorname{SPr}(k))}(G ., j(\mathcal{F})) .
$$

Similarly we have an inclusion

$$
i: \operatorname{Alg}_{k} \hookrightarrow \operatorname{Alg}_{k}^{\Delta}
$$

sending an algebra $B$ to the constant cosimplicial algebra

$$
[n] \mapsto B .
$$

The functor $i$ has a right adjoint sending $A$ to $H^{0}(A)$. If we give $\operatorname{Alg}_{k}$ a model category structure in which fibrations are epimorphisms, equivalences are isomorphisms, and cofibrations are inclusions, then $i$ is a right Quillen functor. Note also that

$$
A \mapsto H^{0}(A)
$$

preserves arbitrary equivalences. From this we obtain a natural isomorphism

$$
[B, A]_{\operatorname{Ho}\left(\operatorname{Alg}_{k}^{\Delta}\right)} \simeq \operatorname{Hom}_{k}\left(B, H^{0}(A)\right),
$$

for any $B \in \operatorname{Alg}_{k}$ and $A \in \operatorname{Alg}_{k}^{\Delta}$.

Suppose $S$ is a $k$-algebra such that $\operatorname{Spec}(S)$ represents the sheaf $\pi_{0}^{\text {sh }}(\mathbb{R} \operatorname{Spec}(A))$. Then for any $k$-algebra $T$ we have canonical isomorphisms

$$
\begin{aligned}
& \operatorname{Hom}_{k}(T, S) \simeq \operatorname{Hom}_{\mathrm{Aff}_{k}}(\operatorname{Spec}(S), \operatorname{Spec}(T)) \\
& \simeq \operatorname{Hom}_{\operatorname{Sh}(k)}(\mathcal{F}, \operatorname{Spec}(T)) \\
& \simeq[\mathbb{R} \operatorname{Spec}(A), \operatorname{Spec}(T)]_{\operatorname{Ho}(\operatorname{SPr}(k))} \text { by (6.7.1) } \\
& \simeq[T, A]_{\mathrm{Ho}\left(\mathrm{Alg}_{k}\right)} \quad \text { by }[\mathrm{To}, 2.2 .3] \\
& \simeq \operatorname{Hom}_{k}\left(T, H^{0}(A)\right) \text { by (6.7.2). }
\end{aligned}
$$


6.8. Let $x, y: A \rightarrow k$ be an augmented object of $\operatorname{Alg}_{k}^{\Delta}$ with associated augmented differential graded algebra $D$. Suppose that there exists a field $k_{0}$ and a pointed object $x_{0}, y_{0}: A_{0} \rightarrow k_{0}$ of $\operatorname{Alg}_{k}^{\Delta}$ such that $(A, x, y)$ is obtained by base change along a morphism $k_{0} \rightarrow k$ from $\left(A_{0}, x_{0}, y_{0}\right)$. Then the sheaf $\pi_{0}^{\text {sh }}\left(P_{x, y}\right)$ associated to the presheaf

$$
R \mapsto \pi_{0}\left(P_{x, y}(R)\right)
$$

is representable, as this sheaf is a torsor under the sheaf associated to the presheaf

$$
R \mapsto \pi_{1}(\mathbb{R} \operatorname{Spec}(A)(R), x)
$$

which is representable by [To, 2.4.5].

We conclude that the sheaf $\pi_{0}^{\mathrm{sh}}\left(P_{x, y}\right)$ is represented by the spectrum of the ring

$$
H^{0}(E),
$$

where $E$ is the homotopy colimit is taken in the category $\operatorname{dga}_{k}^{\geq 0}$ of the diagram

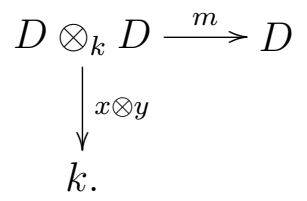

\section{Constructing COfibrant Representatives When $k$ is a Field.}

7.1. In general, cofibrations in $\operatorname{dga}_{k}^{\geq 0}$ are mysterious. However, in the case when $k$ is a field of characteristic 0 , which we assume for the remainder of this section, one can construction cofibrant representatives using the theory of minimal models (see for example [BG]). For the convenience of the reader we recall the argument. The main result of this section is the following proposition.

Proposition 7.2. If $k$ is a field and $a: A \rightarrow B$ is a morphism in $\operatorname{dga}_{k}^{\geq 0}$ with $H^{0}(A)=$ $H^{0}(B)=k$, then there exists a factorization of a in $\operatorname{dga}_{k}^{\geq 0}$

$$
A \stackrel{a^{\prime}}{\longrightarrow} A^{\prime} \stackrel{b}{\longrightarrow} B
$$

where $a^{\prime}$ is a cofibration in $\mathrm{dga}_{k}$ and $b$ is a trivial fibration.

Proof. The proof occupies the remainder of this section.

Lemma 7.3. Let $A \in \mathrm{dga}_{k}^{\geq 0}$ be an object, let $V$ be a k-vector space, and let $\alpha: V \rightarrow Z_{A}^{q}$ be a map to the closed elements $Z_{A}^{q}$ in $A^{q}$. If the induced map

$$
V \rightarrow H^{q}(A)
$$

is injective, then the natural map

$$
A \rightarrow A\langle V[-q], \alpha\rangle
$$

induces an isomorphism

$$
H^{i}(A) \rightarrow H^{i}(A\langle V[-q], \alpha\rangle)
$$

for $i<q$. 
Proof. Let us first consider the case $q=1$. In this case

$$
(A\langle V[-q], \alpha\rangle)^{0}=A^{0} \otimes_{k} \operatorname{Sym}^{\cdot} V .
$$

For $s \geq 0$, let $F^{s} \subset \operatorname{Sym}^{\prime} V$ be the $k$-subspace generated by monomials of degree $\leq s$. Then $F^{s-1} \subset F^{s}$, and the differential on $A^{0}\langle V[-q], \alpha\rangle$ sends $A^{0} \otimes F^{s}$ to $A^{1} \otimes F^{s}$. In particular, if we write $\operatorname{gr}_{F}^{s}$ for $F^{s} / F^{s-1}$, then the differential on $A\langle V[-q], \alpha\rangle$ induces a map

$$
A^{0} \otimes \operatorname{gr}_{F}^{s} \rightarrow A^{1} \otimes \operatorname{gr}^{s} F \text {. }
$$

By construction this map is equal to $d_{A} \otimes 1$.

Let $z \in A^{0} \otimes \operatorname{Sym}^{\prime} V$ be a closed element, and write

$$
z=z_{0}+z_{1}+\cdots+z_{n}
$$

where $z_{i} \in A^{0} \otimes \operatorname{Sym}^{i} V$. Let $\left[z_{n}\right] \in A^{0} \otimes \operatorname{gr}_{F}^{n}$ be the image of $z_{n}$. Then $\left(d_{A} \otimes 1\right)\left[z_{n}\right]=0$ since $z$ is closed. The assumption that $H^{0}(A)=k$ then implies that $z_{n} \in k \otimes_{k} \operatorname{Sym}^{n} V \subset A^{0} \otimes \operatorname{Sym}^{n} V$. Let

$$
\partial: \operatorname{Sym}^{n} V \rightarrow V \otimes \operatorname{Sym}^{n-1}(V)
$$

be the map sending

$$
v_{1} \otimes \cdots \otimes v_{n} \mapsto \sum_{i=1}^{n} v_{i} \otimes\left(v_{1} \otimes \cdots \otimes \hat{v}_{i} \otimes \cdots \otimes v_{n}\right) .
$$

Then it follows that the image under the differential of $z_{n}$ in $A^{1} \otimes \operatorname{Sym} V$ is equal to the image of $\partial\left(z_{n}\right)$ under the inclusion

$$
V \otimes \operatorname{Sym}^{n-1} V \hookrightarrow A^{1} \otimes \operatorname{Sym}^{\cdot} V
$$

In particular, $\partial\left(z_{n}\right) \in A^{1} \otimes \operatorname{Sym}^{n-1} V$. Furthermore, since $z$ is closed we must have $\left[\partial\left(z_{n}\right)\right]=$ $\left[-\left(d_{a} \otimes 1\right)\left[z_{n-1}\right]\right]$ in

$$
A^{1} \otimes \operatorname{gr}_{F}^{n-1}
$$

View $A^{*} \otimes \operatorname{gr}_{F}^{n-1}$ as a complex with differential $d_{A} \otimes 1$. Then

$$
H^{i}\left(A \otimes \operatorname{gr}_{F}^{n-1}\right) \simeq H^{i}\left(A^{\cdot}\right) \otimes \operatorname{gr}_{F}^{n-1}
$$

In particular, since $V \rightarrow H^{1}(A)$ is injective, we must have $\partial\left(z_{n}\right)=0$. This is impossible if $n>0$. We conclude that $z \in A^{0}$, and therefore is in $k$.

Next consider the case $q>1$. Note first of all that the map

$$
A^{i} \rightarrow(A\langle V[-q], \alpha\rangle)^{i}
$$

is an isomorphism for $i<q-1$ and injective for all $i$. It follows that the map

$$
H^{i}(A) \rightarrow H^{i}(A\langle V[-q], \alpha\rangle)
$$

is an isomorphism for $i<q-1$, and injective for $i=q-1$. It remains to verify the surjectivity on $H^{q-1}$. For this observe that

$$
(A\langle V[-q], \alpha\rangle)^{q-1}=A^{q-1} \oplus A^{0} \otimes_{k} V
$$

and

$$
(A\langle V[-q], \alpha\rangle)^{q}= \begin{cases}A^{q} \oplus A^{1} \otimes V & \text { if } q>2 \\ A^{q} \oplus A^{1} \otimes V \oplus A^{0} \otimes \bigwedge^{2} V & \text { if } q=2 .\end{cases}
$$


Let $(z, w) \in A^{q-1} \oplus A^{0} \otimes V$ be a closed element. The image under the differential (the first component in the caes $q=2$ ) is then equal to

$$
\left(d_{A} z+\alpha(w),\left(d_{A} \otimes 1\right)(w)\right) \in A^{q} \oplus A^{1} \otimes V,
$$

where we write $\alpha(w)$ for the image of $w$ under the composite

$$
A^{0} \otimes V \stackrel{1 \otimes \alpha}{\longrightarrow} A^{0} \otimes A^{q} \longrightarrow A^{q}
$$

where the second map is multiplication. We conclude that $w$ is in the kernel of the map

$$
\left(d_{A} \otimes 1\right): A^{0} \otimes V \rightarrow A^{1} \otimes V,
$$

and therefore since $H^{0}(A)=k$ we conclude that $w \in V=k \otimes V \subset A^{0} \otimes V$. Therefore the image of $w$ under

$$
\alpha: V \rightarrow A^{1}
$$

is a boundary. Since $V \rightarrow H^{1}(A)$ is injective this implies that $w=0$. Therefore the map

$$
H^{q-1}(A) \rightarrow H^{q-1}(A\langle V[-q], \alpha\rangle)
$$

is surjective.

We now construct inductively a sequence of morphisms in $\operatorname{dga}_{\bar{k}}^{\geq 0}$

$$
A=A_{-1}^{\prime} \rightarrow A_{0}^{\prime} \rightarrow A_{1}^{\prime} \rightarrow \cdots,
$$

with compatible morphisms $\rho_{q}: A_{q}^{\prime} \rightarrow B$ such that the composite

$$
A \longrightarrow A_{q}^{\prime} \stackrel{\rho_{q}}{\longrightarrow} B
$$

is equal to $a$, and such that the following hold:

(i) For all $q$ the map

is a cofibration in $\operatorname{dga}_{k}$.

$$
A_{q}^{\prime} \rightarrow A_{q+1}^{\prime}
$$

(ii) The map

$$
H^{i}\left(A_{q}^{\prime}\right) \rightarrow H^{i}(B)
$$

induced by $\rho_{q}$, is an isomorphism for $i \leq q$ and an injection for $i=q+1$.

For $q=-1$ we take $A_{-1}^{\prime}=A$.

So assume

$$
A \longrightarrow A_{q}^{\prime} \stackrel{\rho_{q}}{\longrightarrow} B
$$

has been constructed.

First choose a $k$-subspace $j: W \subset Z_{B}^{q+1}$ such that the induced map

$$
H^{q+1}\left(A_{q}^{\prime}\right) \oplus W \rightarrow H^{q+1}(B)
$$

is an isomorphism. Let $\alpha: W \rightarrow A^{q+2}$ be the zero map, and set

$$
C_{q+1}:=A_{q}^{\prime}\langle W[-q-2], \alpha\rangle .
$$

We then have a factorization of $\rho_{q}$

$$
A_{q}^{\prime} \longrightarrow C_{q+1} \longrightarrow B
$$


where the second map is induced by $j$. Furthermore the induced map

$$
H^{i}\left(C_{q+1}\right) \rightarrow H^{i}(B)
$$

is an isomorphism for $i \leq q+1$. It then remains to arrange for the map on $H^{q+2}$ to be injective.

For this let $V \subset H^{q+2}\left(C_{q+1}\right)$ be the kernel of the map to $H^{q+2}(B)$, and choose a lifting $\alpha: V \rightarrow Z_{C_{q+1}}^{q+2}$. We can then find a factorization of $C_{q+1} \rightarrow B$ as

$$
C_{q+1} \rightarrow C_{q+1}\langle V[-q-2], \alpha\rangle \rightarrow B
$$

By 7.3, the map $H^{i}\left(C_{q+1}\right) \rightarrow H^{i}\left(C_{q+1}\langle V[-q-2], \alpha\rangle\right)$ is an isomorphism for $i \leq q+1$, and for $i=q+2$ the map sends $V$ to zero. Repeating this construction inductively we get a sequence of cofibrations in dga $_{k}$

$$
C_{q+1}=D_{0} \rightarrow D_{1} \rightarrow D_{2} \rightarrow \cdots,
$$

together with compatible maps $\sigma_{t}: D_{t} \rightarrow B$ such that the following hold:

(1) For every $t$, the map $H^{i}\left(D_{t}\right) \rightarrow H^{i}\left(D_{t+1}\right)$ is an isomorphism for $i \leq q+1$.

(2) The map $H^{q+2}\left(D_{t}\right) \rightarrow H^{q+2}\left(D_{t+1}\right)$ sends the kernel of $H^{q+2}\left(D_{t}\right) \rightarrow H^{q+2}(B)$ to zero.

We now define $A_{q+1}^{\prime}$ to be $\lim _{\longrightarrow} D_{t}$ with the map $\rho_{q+1}: A_{q+1}^{\prime} \rightarrow B$ the map defined by the $\sigma_{t}$.

The main application for us of this result is the following:

Corollary 7.4. Let $k$ be a field of characteristic 0 , and let $R$ be a $k$-algebra. Let $A \in \operatorname{dga} \geq_{\bar{R}}^{\geq 0}$ be an object with two augmentations $x, y: A \rightarrow R$, and consider the following diagram, which we shall denote $\mathscr{D}$,

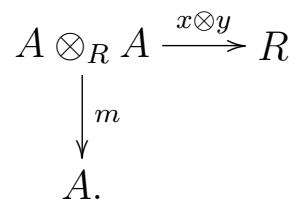

Suppose further that $(A, x, y)$ is obtained by base change from an object $A_{0} \in \operatorname{dga}_{\bar{k}}^{\geq 0}$ with two augmentations $x_{0}, y_{0}: A_{0} \rightarrow k$, and such that $H^{0}\left(A_{0}\right)=k$. Then the natural map defined in 5.3.2

$$
\operatorname{hocolim}(\mathscr{D}) \rightarrow J\left(\operatorname{hocolim}^{\geq 0}(\mathscr{D})\right)
$$

is an isomorphism. In particular, if $\pi_{0}\left(P_{x, y}\right)$ denotes the functor

$$
\operatorname{Alg}_{R} \rightarrow \text { Set, } S \mapsto \pi_{0}\left(P_{x, y}(S)\right),
$$

where $P_{x, y}$ is the path space of the augmented cosimplicial algebra associated to $(A, x, y)$, then there is a canonical isomorphism

$$
\pi_{0}\left(P_{x, y}\right) \simeq \operatorname{Spec}\left(H^{0}(B(A, x, y))\right)
$$

Proof. Note that we also have $H^{0}\left(A_{0} \otimes_{k} A_{0}\right)=k$. It then follows from 7.2 that the assumptions of 5.4 are satisfied for the diagram $\mathscr{D}$. 


\section{Application to p-ADiC Hodge theory}

Let $(X, D) / V$ be as in 1.1, and let $x, y \in X^{\circ}(V)$ be two points. Assume further that $X$ is geometrically connected. We can then consider various differential graded algebras associated to $X^{\circ}$.

8.1. De Rham realization. Let $U . \rightarrow X_{K}$ be an étale hypercover with each $U_{n}$ affine. We then obtain a cosimplicial differential graded algebra $D R(U$.) by

$$
[n] \mapsto \Gamma\left(U_{n}, \Omega_{U_{n} / K}(\log )\right),
$$

where $\Omega_{U_{n} / K}(\log )$ denotes the de Rham complex with log poles along the preimage of $D$. We let $A_{\mathrm{DR}}$ denote the differential graded $K$-algebra obtained by applying the functor of Thom-Sullivan cochains to this cosimplicial differential graded algebra. The cohomology of $A_{\mathrm{DR}}$ is the de Rham cohomology of $X_{K}^{o} / K$.

Write also $x, y \in X(K)^{o}$ for the two $K$-valued points induced by the given $V$-valued points. For suitable choice of the hypercover $U$, we can lift these points to morphisms

$$
x, y: \operatorname{Spec}(K) \rightarrow U \text {.. }
$$

For example we can take the hypercovering associated to a Zariski covering of $X_{K}$. These two points then define augmentations

$$
x, y: A_{\mathrm{DR}} \rightarrow K .
$$

Let $P_{x, y}^{\mathrm{DR}}$ be the space of paths obtained as in section 6 from $\left(A_{\mathrm{DR}}, x, y\right)$ by first converting $A_{\mathrm{DR}}$ into a cosimplicial algebra with augmentations, then applying the functor $\mathbb{R} S p e c$, and then taking $\pi_{0}$ of the space of paths between $x$ and $y$. It follows from $[\mathrm{Ol}, 8.30]$ that $P_{x, y}^{\mathrm{DR}}$ has the following interpretation.

Let $\mathscr{C}_{\mathrm{DR}}$ denote the category of unipotent modules with integrable connection on $X_{K}^{o}$. It is a Tannakian category. The two points $x, y \in X^{o}(K)$ defines fiber functors

$$
\omega_{x}, \omega_{y}: \mathscr{C}_{\mathrm{DR}} \rightarrow \operatorname{Vec}_{K}
$$

and the scheme $P_{x, y}^{\mathrm{DR}}$ represents the functor

$$
(K \text {-algebras }) \rightarrow \text { Set }, \quad R \mapsto\left\{\text { set of isomorphisms of fiber functors } \omega_{x} \otimes R \rightarrow \omega_{y} \otimes R\right\} .
$$

8.2. The bar complex $B\left(A_{\mathrm{DR}}, x, y\right)$ can be described as follows. By 4.8 , it is given by the total complex associated to the simplicial differential graded algebra

$$
T\left(A_{\mathrm{DR}}, x, y\right):[n] \mapsto \underbrace{A_{\mathrm{DR}} \otimes \cdots \otimes A_{\mathrm{DR}}}_{n}
$$

described in 4.7. This simplicial differential graded algebra can also be described as follows. Let $(X, x, y)^{\cdot}$ be the cosimplicial scheme

$$
[n] \mapsto X^{n}
$$

with transition maps given by

$$
\begin{gathered}
d^{0}\left(x_{1}, \ldots, x_{n}\right)=\left(x_{1}, \ldots, x_{n}, x\right), d^{n+1}\left(x_{1}, \ldots, x_{n}\right)=\left(y, x_{1}, \ldots, x_{n}\right), \\
d^{i}\left(x_{1}, \ldots, x_{n}\right)=\left(x_{1}, \ldots, x_{n-i+1}, x_{n-i+1}, \ldots, x_{n}\right), \quad 0<i<n+1,
\end{gathered}
$$

and the codegeneracy operators defined by the projections. 
We can then consider the de Rham complex of $(X, x, y)_{K}$. This is again computed using a hypercovering $U$. $\rightarrow X$ as above. Namely, we can also consider the cosimplicial simplicial scheme

$$
[n] \mapsto U^{n} .
$$

Taking the de Rham complex with log poles of each $U_{m}^{n}$ defines a functor

$$
\Delta^{o} \times \Delta \mapsto \operatorname{dga}_{K}, \quad([n],[m]) \mapsto \Gamma\left(U_{m, K}^{n}, \Omega_{U_{m, K}^{n} / K}\right)
$$

Applying the functor of Thom-Sullivan cochains in the cosimplicial direction we obtain a simplicial differential graded algebra $T(U ., x, y)$. Note that there is a natural morphism

$$
T\left(A_{\mathrm{DR}}, x, y\right) \rightarrow T(U ., x, y)
$$

which is a level-wise equivalence. We therefore obtain a canonical isomorphism

$$
P_{x, y}^{\mathrm{DR}} \simeq \operatorname{Spec}\left(H^{0}(\operatorname{tot}(T(U,, x, y)))\right) .
$$

8.3. The advantage of considering the simplicial scheme $U$. is that each differential graded algebra

$$
\Gamma\left(U_{m, K}^{n}, \Omega_{U_{m, K}^{n} / K}\right)
$$

is naturally an object of fdga ${ }_{K}$, with filtration given by the Hodge filtration. Applying the functor of filtered Thom-Sullivan cochains, the simplicial differential graded algebra $T(U ., x, y)$ is naturally a simplicial object in $\mathrm{fdga}_{K}$. We conclude that

$$
H^{0}(\operatorname{tot}(T(U,, x, y))) \simeq \mathscr{O}_{P_{x, y}^{\mathrm{DR}}}
$$

has a natural filtration, which we call the Hodge filtration.

Remark 8.4. A straightforward verification, which we leave to the reader, shows that the Hodge filtration on $\mathscr{O}_{P_{x, y}^{\mathrm{DR}}}$ is independent of the choices in the above construction.

8.5. Étale realization. Here we again start with an étale hypercover $U . \rightarrow X_{K}$ such that each $U_{\bar{K}}^{o}$ is a $K(\pi, 1)$ (see $[\mathrm{Ol} 2, \S 5.1]$ for what this means). Given a collection $E$ of geometric generic points of $X_{\bar{K}}$, the construction in [Ol, §5] defines a cosimplicial differential graded algebra

$$
[n] \mapsto G C\left(U_{n, \bar{K}}^{o}, E\right) .
$$

Applying the functor of Thom-Sullivan cochains we obtain a differential graded algebra $A_{\text {et }} \in$ $\operatorname{dga}_{\mathbb{Q}_{p}}$. Moreover, there is a natural action of $\mathrm{Gal}_{\bar{K} / K}$ on the image of this algebra in $\operatorname{Ho}\left(\operatorname{dga}_{\mathbb{Q}_{p}}\right)$

Two points $x, y: \operatorname{Spec}(K) \rightarrow X^{o}$ define augmentations $A_{\text {et }} \rightarrow \mathbb{Q}_{p}$. Let $P_{x, y}^{\text {et }}$ be the affine scheme associated to $\left(A_{\mathrm{et}}, x, y\right)$ as in section 4 .

The Tannakian interpretation of $P_{x, y}^{\text {et }}$ is the following. Let $\mathscr{C}_{\text {et }}$ denote the category of unipotent sheaves of $\mathbb{Q}_{p}$-spaces on $X_{\bar{K}}^{o}$. This is a Tannakian category and the two points $x$ and $y$ define fiber functors

$$
\omega_{x}, \omega_{y}: \mathscr{C}_{\text {et }} \rightarrow \operatorname{Vec}_{\mathbb{Q}_{p}}
$$

By $[\mathrm{Ol}, 8.30]$, the scheme $P_{x, y}^{\text {et }}$ represents the functor

$\left(\mathbb{Q}_{p}\right.$-algebras $) \rightarrow$ Set, $R \mapsto\left\{\right.$ set of isomorphisms of fiber functors $\left.\omega_{x} \otimes R \rightarrow \omega_{y} \otimes R\right\}$.

On the other hand, by our earlier discussion there is a canonical isomorphism

$$
P_{x, y}^{\mathrm{et}} \simeq \operatorname{Spec}\left(H^{0}\left(B\left(A_{\mathrm{et}}, x, y\right)\right)\right) \text {. }
$$


8.6. We would like to again relate this to the cosimplicial scheme $\left(X_{\bar{K}}^{o}, x, y\right)^{\prime}$, and then consider the p-adic comparison isomorphism for the cosimplicial scheme $(X, x, y)$. This, however, requires some care with base points so let us begin by making some general observations.

Let

$$
f: Y:=\operatorname{Spec}(B) \rightarrow X:=\operatorname{Spec}(A)
$$

be a morphism of geometrically connected smooth $V$-schemes, and assume given divisors $D_{Y} \subset Y$ and $D_{X} \subset X$ with normal crossings relative to $V$ such that $f^{-1}\left(D_{X}\right) \subset D_{Y}$. As usual we set

$$
X^{o}:=X-D_{X}, \quad Y^{o}:=Y-D_{Y} .
$$

Fix also geometric generic points

$$
\eta_{X}: \operatorname{Spec}\left(k\left(\eta_{X}\right)\right) \rightarrow X_{\bar{K}}, \quad \eta_{Y}: \operatorname{Spec}\left(k\left(\eta_{Y}\right)\right) \rightarrow Y_{\bar{K}}
$$

Let $A \subset \bar{A}$ (resp. $B \subset \bar{B}$ ) be the integral closure of $A$ (resp. $B$ ) in the maximal field extension $k(X) \subset L \subset k\left(\eta_{X}\right)\left(\right.$ resp. $k(Y) \subset L \subset k\left(\eta_{Y}\right)$ ) such that the normalization of $X_{\bar{K}}^{o}$ (resp. $\left.Y_{\bar{K}}^{o}\right)$ in $L$ is étale over $X_{\bar{K}}^{o}\left(\operatorname{resp} . Y_{K}^{o}\right)$.

Consider the fiber functors

$$
\omega_{X}: \operatorname{Fet}\left(X_{\bar{K}}^{o}\right) \rightarrow \text { Set, } \omega_{Y}: \operatorname{Fet}\left(Y_{\bar{K}}^{o}\right) \rightarrow \text { Set }
$$

defined by the geometric points $\eta_{X}$ and $\eta_{Y}$ respectively.

Lemma 8.7. There is a canonical bijection between morphisms $\bar{f}: \operatorname{Spec}(\bar{B}) \rightarrow \operatorname{Spec}(\bar{A})$ such that the diagram

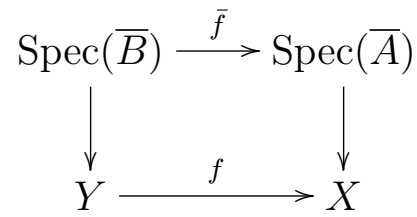

commutes, and isomorphisms $\sigma$ between the two fiber functors

$$
\omega_{X}, \omega_{Y} \circ f^{*}: \operatorname{Fet}\left(X_{\bar{K}}^{o}\right) \rightarrow \text { Set. }
$$

Proof. Let $A^{\prime} \subset k\left(\eta_{X}\right)$ (resp. $B^{\prime} \subset k\left(\eta_{Y}\right)$ ) be the coordinate ring of the integral closure of $X_{\bar{K}}^{o}$ (resp. $Y_{\bar{K}}^{o}$ ) in the maximal extension $L \subset k\left(\eta_{X}\right)$ (resp. $L \subset k\left(\eta_{Y}\right)$ ) such that the normalization is étale over $X_{\bar{K}}^{o}$ (resp. $\left.Y_{\bar{K}}^{o}\right)$. We have $\bar{A} \subset A^{\prime}$ and $\bar{B} \subset B^{\prime}$. Then $\operatorname{Spec}\left(A^{\prime}\right)$ $\left(\right.$ resp. $\left.\operatorname{Spec}\left(B^{\prime}\right)\right)$ is the universal covering of $X_{\bar{K}}^{o}$ (resp. $Y_{\bar{K}}^{o}$ ). We therefore have a canonical bijection between the set of isomorphisms of fiber functors $\sigma$ between the two functors in 8.7.2, and dotted arrows filling in the following diagram

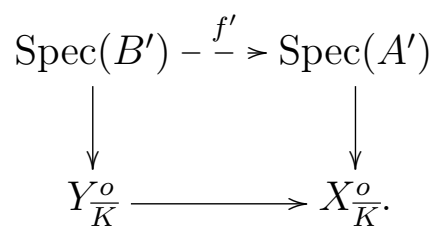

Thus it remains to observe that there is a canonical bijection between the set of dotted arrows $f^{\prime}$ and the set of arrows $\bar{f}$ filling in the diagram 8.7.1. 
Remark 8.8. Note that the bijection in 8.7 is compatible with the natural actions of the fundamental groups. Namely, by construction there is a natural action of $\pi_{1}\left(X_{\bar{K}}^{o}, \omega_{X}\right)$ (resp. $\left.\pi_{1}\left(Y_{\bar{K}}^{o}, \omega_{Y}\right)\right)$ on $\operatorname{Spec}(\bar{A})$ over $X$ (resp. $\operatorname{Spec}(\bar{B})$ over $\left.Y\right)$. Given an isomorphism $\sigma$ between the fiber functors in 8.7.2 we obtain a morphism of fundamental groups

$$
\sigma_{*}: \pi_{1}\left(Y_{\bar{K}}^{o}, \omega_{Y}\right) \rightarrow \pi_{1}\left(X_{\bar{K}}^{o}, \omega_{X}\right)
$$

and by the construction the morphism $\bar{f}$ corresponding to $\sigma$ is compatible with the group actions.

8.9. Consider now a functor

$$
U^{(\cdot)}: \Delta \times \Delta^{o} \rightarrow(V \text {-schemes }), \quad([n],[m]) \mapsto U_{m}^{(n)},
$$

such that each $U_{m}^{(n)}$ is a finite disjoint union of affine smooth $V$-schemes with connected geometric generic fiber.

Consider also a second functor

$$
E^{(\cdot)}: \Delta \times \Delta^{o} \rightarrow(\bar{K} \text {-schemes })
$$

such that for every $n$ and $m$ the scheme $E_{n}^{(m)}$ is a finite disjoint union of separably closed fields. Suppose further given a morphism of simplicial cosimplicial schemes

$$
E_{\cdot}^{(\cdot)} \rightarrow U_{\cdot, \bar{K}}^{(\cdot)}
$$

such that for every $n$ and $m$ the map

$$
E_{n}^{(m)} \rightarrow U_{n, \bar{K}}^{(m)}
$$

is a family of (not necessarily generic) geometric points of $U_{n, \bar{K}}^{(m)}$, in the sense of [Ol, 5.13].

Choose for every $n$ and $m$, and for each connected component $\Gamma$ of $U_{n, \bar{K}}^{(m)}$ a geometric generic point

$$
\eta_{n, m, \Gamma}: \operatorname{Spec}\left(\Omega_{n, m, \Gamma}\right) \rightarrow \Gamma_{\bar{K}} \subset U_{n, \bar{K}}^{(m)}
$$

Choose furthermore for every $e \in E_{n}^{(m)}$ mapping to $\Gamma_{e}$ an isomorphism

$$
\iota_{e}: \omega_{e} \rightarrow \omega_{\eta_{n, m, \Gamma}}
$$

between the two fiber functors

$$
\operatorname{FEt}\left(\Gamma_{e, \bar{K}}\right) \rightarrow \text { Set. }
$$

Let $\pi_{n, m, \Gamma_{e}}$ denote the fundamental group of $\Gamma_{e, \bar{K}}$ with respect to the base point $\eta_{n, m, \Gamma_{e}}$. Define

$$
G C\left(U_{n, \bar{K}}^{(m)}, \mathbb{Q}_{p}\right):=\oplus_{e \in E_{n}^{(m)}} G C\left(U_{n, \bar{K}}^{(m)}, \eta_{n, m, \Gamma_{e}}, \mathbb{Q}_{p}\right) .
$$

This is a differential graded $\mathbb{Q}_{p}$-algebra. As $n$ and $m$ vary these algebras form a simplicial cosimplicial algebra as follows.

Consider morphisms in $\Delta$

$$
\delta_{1}:\left[n^{\prime}\right] \rightarrow[n], \quad \delta_{2}:[m] \rightarrow\left[m^{\prime}\right]
$$

defining a morphism

$$
\delta: U_{n}^{(m)} \rightarrow U_{n^{\prime}}^{\left(m^{\prime}\right)}
$$


For any $e \in E_{n}^{(m)}$ with image $\delta(e) \in E_{n^{\prime}}^{\left(m^{\prime}\right)}$ we then have an isomorphism of fiber functors

$$
\sigma_{e}: \omega_{\delta(e)} \rightarrow \omega_{e} \circ \delta^{*} .
$$

Using the isomorphisms $\iota_{e}$ and $\iota_{\delta(e)}$ this defines an isomorphism of fiber functors

$$
\sigma_{e}: \omega_{n^{\prime}, m^{\prime}, \Gamma_{\delta(e)}} \rightarrow \omega_{n, m, \Gamma_{e}} \circ \delta^{*} .
$$

This in turn induces a morphism of differential graded algebras

$$
G C\left(U_{n^{\prime}, \bar{K}}^{\left(m^{\prime}\right)}, \eta_{n^{\prime}, m^{\prime}, \Gamma_{\delta(e)}}, \mathbb{Q}_{p}\right) \rightarrow G C\left(U_{n, \bar{K}}^{(m)}, \eta_{n, m, \Gamma_{e}}, \mathbb{Q}_{p}\right)
$$

Putting all these together we obtain a morphism of differential graded algebras

$$
G C\left(U_{n^{\prime}, \bar{K}}^{\left(m^{\prime}\right)}, \mathbb{Q}_{p}\right) \rightarrow G C\left(U_{n, \bar{K}}^{(m)}, \mathbb{Q}_{p}\right) .
$$

It follows from the construction that these maps are compatible with composition so we obtain a cosimplicial simplicial differential graded algebra. Applying the functor of Thom-Sullivan cochains we obtain a simplicial differential graded algebra, which we shall denote by

$$
T\left(U^{(\cdot)}, \mathbb{Q}_{p}\right)
$$

8.10. The advantage of this construction is that it works well also with other coefficient rings, such as Fontaine's ring $A_{\text {cris }}$.

Let $(X, D) / V$ be as in 1.1, and let $x, y: \operatorname{Spec}(V) \rightarrow X^{o}$ be two points. Choose an étale hypercovering $U . \rightarrow X$ with each $U_{n}$ very small, in the sense of [Ol, 6.1]. Choose furthermore liftings

$$
\operatorname{Spec}(V) \rightarrow U
$$

of $x$ and $y$ (this is possible as we can take $U_{0}$ as obtained from a Zariski covering of $X$ ). We then obtain a functor

$$
(U ., x, y)^{\cdot}: \Delta \rightarrow(\text { simplicial } \log \text { schemes })
$$

by

$$
[n] \mapsto U_{.}^{n},
$$

with transition maps defined as in 8.2.1.

We can also apply this construction with $\operatorname{Spec}(V) \coprod \operatorname{Spec}(V)$ with the two inclusions of $\operatorname{Spec}(V)$. Let

$$
\Sigma: \Delta \rightarrow(\text { schemes })
$$

be the resulting functor, so that

$$
\Sigma^{n}=\coprod_{i=0}^{n} \operatorname{Spec}(V)
$$

There is a natural map

$$
\Sigma \rightarrow(X, x, y)^{\cdot}
$$

induced by $x$ and $y$. For $[n],[m] \in \Delta$ define $E_{n}^{m}$ to be

$$
\coprod_{\operatorname{Hom}_{(X, x, y)^{m}}\left(\Sigma^{m},\left(U_{n}, x, y\right)^{m}\right)} \Sigma^{m} .
$$

The $E_{n}^{m}$ define a simplicial cosimplicial scheme, there is a natural morphism

$$
E \text {. } \rightarrow(U ., x, y)
$$


and each $E_{n}^{m}$ is isomorphic to a finite disjoint union of copies of $\operatorname{Spec}(V)$. Furthermore, the induced map

$$
E_{\cdot, \bar{K}}^{\cdot} \rightarrow\left(U_{\cdot, \bar{K}}^{o}, x, y\right)^{\cdot}
$$

is a family of geometric points. Choosing generic base points

$$
\eta_{n, m, \Gamma}: \operatorname{Spec}\left(\Omega_{n, m, \Gamma}\right) \rightarrow\left(U_{n, \bar{K}}^{o}, x, y\right)^{m}
$$

and isomorphisms $\iota_{e}$ as in 8.9, we therefore obtain a simplicial differential graded algebra

$$
T\left(\left(U_{\cdot, \bar{K}}^{o}, x, y\right)^{\cdot}, \mathbb{Q}_{p}\right)
$$

and a natural equivalence

$$
B\left(A_{\mathrm{et}}, x, y\right) \rightarrow \operatorname{tot}\left(T\left(\left(U_{\cdot, \bar{K}}^{o}, x, y\right)^{\cdot}, \mathbb{Q}_{p}\right)\right) .
$$

8.11. Let $\left(U^{\wedge}, x, y\right)^{\cdot}$ be the simplicial cosimplicial space sending $([n],[m]) \in \Delta \times \Delta^{o}$ to the $p$-adic completion of $\left(U_{n}, x, y\right)^{m}$. Note that because $V$ is $p$-adically complete the morphism 8.10.1 lifts uniquely to a morphism

$$
E_{.} \rightarrow\left(U^{\wedge}, x, y\right)^{\cdot}
$$

We can furthermore choose geometric generic points

$$
\hat{\eta}_{n, m, \Gamma}: \operatorname{Spec}\left(\widehat{\Omega}_{n, m, \Gamma}\right) \rightarrow\left(U_{n, \bar{K}}^{\wedge o}, x, y\right)^{m}
$$

extending the $\eta_{n, m, \Gamma}$. We then obtain a simplicial differential graded algebra

$$
T\left(\left(U_{\cdot, K}^{\wedge o}, x, y\right)^{\cdot}, \mathbb{Q}_{p}\right)
$$

and there is a natural map

$$
T\left(\left(U_{\cdot, \bar{K}}^{o}, x, y\right)^{\prime}, \mathbb{Q}_{p}\right) \rightarrow T\left(\left(U_{\cdot, \bar{K}}^{\wedge o}, x, y\right)^{\cdot}, \mathbb{Q}_{p}\right) .
$$

For every $n$ and $m$, we also have the Galois module

$$
\mathrm{B}_{\text {cris }}\left(\left(U_{n}^{\wedge}, x, y\right)^{m}\right) .
$$

By 8.7, the Galois cohomology groups of these modules are functorial and we obtain another simplicial differential graded algebra

$$
T\left(\left(U^{\wedge}, x, y\right)^{\bullet}, \mathrm{B}_{\text {cris }}\left(\left(U^{\wedge}, x, y\right)^{\cdot}\right)\right)
$$

There are natural maps

$$
T\left(\left(U_{\cdot, \bar{K}}^{o}, x, y\right)^{\cdot}, \mathbb{Q}_{p}\right) \rightarrow T\left(\left(U_{\cdot, \bar{K}}^{\wedge o}, x, y\right)^{\cdot}, \mathbb{Q}_{p}\right) \rightarrow T\left(\left(U_{\cdot}^{\wedge}, x, y\right)^{\cdot}, \mathrm{B}_{\mathrm{cris}}\left(\left(U_{\cdot}^{\wedge}, x, y\right)^{\cdot}\right)\right)
$$

8.12. We can also consider the standard resolution $\mathscr{K} \rightarrow \mathbb{R}^{\bullet}$ of the structure sheaf in the convergent topos of $\left(X, M_{X}\right) / K$. By the same reasoning as in [Ol, 6.17] (and see also this reference for the notation), we then get a diagram of simplicial filtered differential graded 
algebras

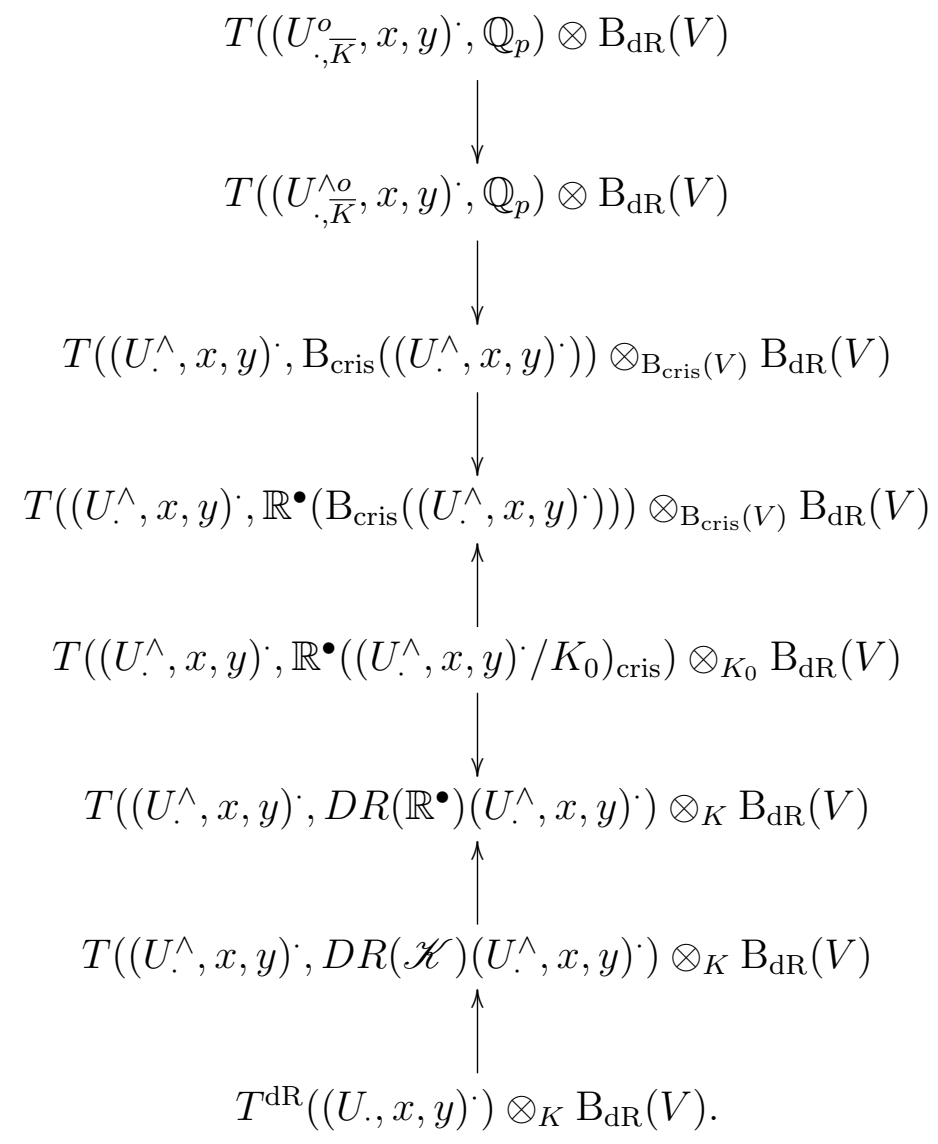

In degree $n$, this sequence of morphisms is the sequence defining the $p$-adic comparison isomorphism for $X^{n}$. In particular, all the maps in the above diagram are filtered quasiisomorphisms, compatible with the action of Galois in the homotopy category. We therefore obtain an isomorphism

$$
H^{0}\left(T^{\mathrm{dR}}(U ., x, y)^{\cdot}\right) \otimes_{K} \mathrm{~B}_{\mathrm{dR}}(V) \simeq H^{0}\left(T\left(\left(U_{\cdot, \bar{K}}^{o}, x, y\right)^{\cdot}, \mathbb{Q}_{p}\right)\right) \otimes \mathrm{B}_{\mathrm{dR}}(V)
$$

compatible with the the Galois action and filtrations. On the other hand, we have a diagram

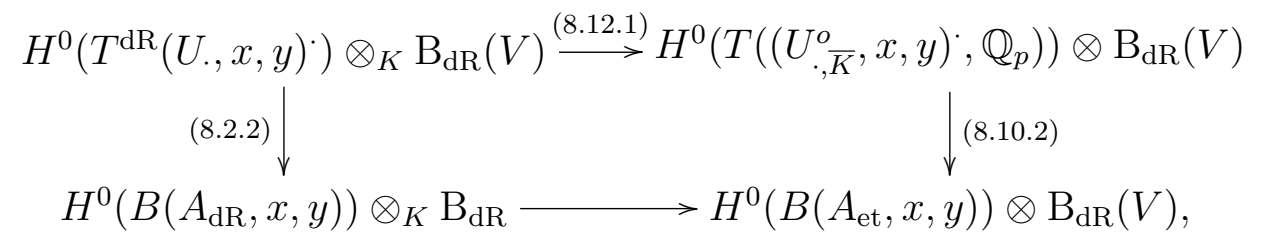

where the bottom horizontal row is the map obtained from the isomorphism in the homotopy category constructed in $[\mathrm{Ol}]$

$$
A_{\mathrm{dR}} \otimes_{K} \mathrm{~B}_{\mathrm{dR}}(V) \simeq A_{\mathrm{et}} \otimes \mathrm{B}_{\mathrm{dR}}(V) .
$$

It follows from the construction that 8.12.2 commutes. In particular, we obtain the following:

Corollary 8.13. The filtration on $\mathscr{O}_{P_{x, y}^{\mathrm{dR}}}$ induced by the isomorphism

$$
\mathscr{O}_{P_{x, y}^{\mathrm{dR}}} \otimes_{K} \mathrm{~B}_{\mathrm{dR}}(V) \simeq \mathscr{O}_{P_{x, y}^{\mathrm{et}}} \otimes_{\mathbb{Q}_{p}} \mathrm{~B}_{\mathrm{dR}}(V)
$$

constructed in [Ol], agrees with the filtration defined using the bar construction in 8.3. 


\section{REFERENCES}

[Be] K. Behrend, Differential grades schemes I: perfect resolving algebras, preprint (2002).

[BG] A.K. Bousfield and V. K. A. M. Gugenheim, On PL de Rham theory and rational homotopy type, Mem. Amer. Math. Soc. 8 (1976), no. 179.

[Bo] A.K. Bousfield and D.M. Kan, Homotopy limits, completions and localizations, Springer Lectures Notes in Math 304 (1972).

[Fo] J.-M. Fontaine, Le corps des périodes p-adiques in "Périodes p-adiques", Astérisque 223 (1994), 59-111.

[Hi] V. Hinich, Homological algebra of homotopy algebras, Communications in algebra 25 (1997), 3291-3323.

[Ho] M. Hovey, Model categories, Mathematical Surveys and Monographs 63, AMS (1990).

[Il] L. Illusie, Complexe cotangent et déformations I, Springer Lecture Notes in Math 239 (1971).

[Ki1] M. Kim, The motivic fundamental group of $\mathbb{P}^{1}-\{0,1, \infty\}$ and the theorem of Siegel, Invent. Math. 161 (2005).

[Ki2] _ The unipotent Albanese map and Selmer varieties for curves, to appear in the Publications of the Research Institute for Mathematical Sciences.

[Ki3] _ , p-adic L-functions and Selmer varieties associated to elliptic curves with complex multiplication, preprint.

[N-A] Navarro-Aznar, Sur la thèorie de Hodge-Deligne, Invent. Math. 90 (1987), 11-76.

[Ol] M. Olsson, Towards nonabelian p-adic Hodge theory, preprint (2008).

[O12] M. Olsson, On Faltings' method of almost étale extensions, to appear in the proceedings of the 2005 AMS Summer Research Institute in Algebraic Geometry,

[Sp] N, Spaltenstein, Resolutions of unbounded complexes, Comp. Math. 65 (1988), 121-154.

[To] B. Toen, Champs affines, Selecta Math. (N.S.) 12 (2006), 39-135.

[Wo] Z. Wojtkowiak, Cosimplicial objects in algebraic geometry, in 'Algebraic K-theory and algebraic topology', edited by Goerss and Jardine, Nato ASI Series (1993), 287-327. 